\title{
Anti-Oxidant Potential Of Metabolites And Synthesised Silver Nanoparticles Of Bacillus Megaterium
}

\author{
Sharmistha Banerjee ${ }^{1}$, Shuchi Kaushik ${ }^{2}$ and Rajesh Singh Tomar ${ }^{3 *}$ \\ ${ }^{1,3}$ Amity Institute of Biotechnology, Amity University Madhya Pradesh, \\ Maharajpura Dang, Gwalior (M.P.), India - 474005 \\ ${ }^{2}$ Madhya Pradesh Forensic Science Lab, Bhopal, India \\ Email:sbanerjee@gwa.amity.edu ${ }^{1}$,shuchi.kaushik2@gmail.com²,rstomar@amity.edu ${ }^{3}$ \\ * Corresponding Author: Prof. (Dr.) R. S. Tomar, Director and Dean (Academics), \\ Amity Institute of Biotechnology, Amity University Madhya Pradesh
}

\begin{abstract}
The main aim of the present study was evaluation of anti-oxidant potential of extracellular and intracellular metabolites of Bacillus megaterium and synthesis of silver nanoparticles from cell free supernatant of unaltered media and mixed media, its characterisation and evaluation of its anti-oxidant potential. The cell free supernatant as well as biomass was collected by centrifugation and stored at $4^{\circ} \mathrm{C}$ for further analysis. Silver Nanoparticles was synthesised in presence of extracellular metabolites of unaltered and mixed media. Antioxidant activity of cell free supernatant, biomass suspension and synthesised silver nanoparticles was evaluated as a measure of percentage DPPH radical scavenging activity, Total Anti-oxidant Capacity and Total Reductive Capability. Appropriate Positive Control was used for comparison and preparation of standard curves. Extracellular metabolites of $B$. megaterium secreted in mixed media have higher antioxidant potential in comparison to that of unaltered media.
\end{abstract}

Keywords- Supernatant; Biomass; DPPH; Metabolites; Reductive

\section{INTRODUCTION}

The major cause of oxidative stress is because of generation of reactive oxygen species (ROS) in excess or when body's natural anti-oxidant is in deficient amount to neutralise the generated free radicals ${ }^{[1-2]}$. Several physical, chemical and environmental factors are responsible for inducing the excessive generation of ROS by living cells. Free radicals are highly unstable molecules, hence interact with variety of bio molecules leading to DNA fragmentation, lipid peroxidation, protein damage and inactivation of certain enzymes thus resulting in various pathological conditions and cell damage. It is also the cause of many major ailments like Cancer, Aging, Diabetes, Cataract, Coronary Heart and inflammatory diseases ${ }^{[3-4]}$.

Anti-oxidants are chemical substances that protect the human body from reactive oxygen species by reducing or preventing oxidation of these molecules. Usually all anti-oxidant molecules have anti-tumor, anti-mutagenic, anti-bacterial and anti-viral activity either in trace or higher amount. Natural antioxidants are present in food, plants and animals. Because of their limited availability, researchers were shifting towards the use of synthetic antioxidants like Butyl hydroxyl anisole, Butyl hydroxyl toluene and Tertbutyl hydroxyquinone, but they were found to be carcinogenic. Hence, the search for novel natural anti-oxidants has made them to look for microbial metabolites. Microbial cells pose a plenty of defense systems. In the present era, there has been an enormous interest in the antioxidant activity of microorganisms and their impact in treatment of diseases. Natural antioxidants like vitamin $\mathrm{C}$, vitamin $\mathrm{E}$, phenolic acids, carotenes, phytoestrogens and phytate extracted from bacteria have been recognized as potential candidates of reducing risks of several diseases.

Study of microbial antioxidants dates back since early 1980s, although many researchers have established correlation between microbes and their antioxidant capacity ${ }^{[5]}$. However, there are only few studies that have been performed on microbes with respect to their anti-oxidant potential and ability to scavenge free radicals ${ }^{[6]}$. Numerous researchers have cited the anti-oxidant potential of many bacteria, for example, Bacillus natto, Bacillus simplex, Bacillus cereus, Bacillus stearothermophilus, Bacillus longum, Micrococcus freudenreichii, Sarcina lutea, Lactobacillus acidophilus, Lactobacillus dextranicum and Thermothrix species ${ }^{[7]}$.

Silver nanoparticles (AgNps) are known to show a vast range of biological activity, like antibacterial ${ }^{[8-}$ ${ }^{9]}$, antifungal ${ }^{[10-11]}$, antiviral ${ }^{[12-14]}$, anti inflammatory ${ }^{[15-16]}$ and thus are catching attention of researchers because of their wider applicability in several biomedical applications. Numerous techniques are available for synthesis of metallic nanoparticles. Examples include, Reduction of metal ions to metal Nanoparticles by electrochemicals, chemicals, radiations, Langmuir-Blodgett, photochemical and biological method ${ }^{[17-22]}$. Among all these available approaches, biological method is the most preferred one for synthesizing nanomaterials. Biological route is less toxic and more environmental friendly. 


\section{MATERIALS AND METHOD}

\subsection{Preparation of microbial extracts}

Microbial extract of Bacillus megaterium containing bioactive metabolites was evaluated for its antioxidative and anti-cancerous activity. Loopful culture of B. megaterium was inoculated in a flask containing $100 \mathrm{ml}$ nutrient broth (unaltered media) and incubated at $37^{\circ} \mathrm{C}$ for 18 hours in order to assess the anti-cancer and anti-oxidant activity of both extracellular and intracellular metabolites. Mixed media was formulated by screening the best carbon and nitrogen sources based on their enhanced antioxidant potential. The composition of unaltered and mixed media is tabulated in Table 1 and Table 2 respectively.

Table 1: Composition of Unaltered Media (UM)

\begin{tabular}{|c|c|c|}
\hline $\begin{array}{c}\text { S. } \\
\text { No. }\end{array}$ & Components & $\begin{array}{r}\text { Concentration } \\
\text { (in gm/L) }\end{array}$ \\
\hline 1. & Peptone & 5 \\
\hline 2. & Beef Extract & 1.5 \\
\hline 3. & $\begin{array}{c}\text { Yeast } \\
\text { Extract }\end{array}$ & 1.5 \\
\hline 5. & $\begin{array}{c}\text { Sodium } \\
\text { Chloride }\end{array}$ \\
\hline 6. & $\begin{array}{r}\text { Volume } \\
\text { make up } \\
\text { with distilled } \\
\text { water }\end{array}$ & $1 \mathrm{~L}$ \\
\hline
\end{tabular}

Table 2: Composition of Mixed Media (MM)

\begin{tabular}{|c|c|c|}
\hline S. No. & Components & $\begin{array}{c}\text { Concentration } \\
\text { (in } \mathbf{g m} / \mathbf{L} \text { ) }\end{array}$ \\
\hline 1. & Potassium Nitrate & 5 \\
\hline 2. & Beef Extract & 1.5 \\
\hline 3. & Dextrose & 1.5 \\
\hline 4. & Sodium Chloride & 5 \\
\hline 5. & $\begin{array}{c}\text { Volume make } \\
\text { up with } \\
\text { distilled water }\end{array}$ & $1 \mathrm{~L}$ \\
\hline
\end{tabular}

2.2 Separation of Extracellular and Intracellular Metabolites

Biomass was harvested and separated from media supernatant by centrifugation at $10,000 \mathrm{rpm}$ for 10 minutes. Both the cell free supernatant containing extracellular metabolites and pellet containing intracellular metabolites was collected. The supernatant was stored at $4^{\circ} \mathrm{C}$ for further activity analysis. Wet weight of pellet was taken and further resuspended in sterile distilled water at a concentration of $1 \mathrm{mg} / \mathrm{ml}$. Several anti-oxidant assays were performed by following the standard protocols with slight modifications.
2.3 Evaluation of anti-oxidant activity of intracellular and extracellular metabolites

The anti-oxidant activity of intracellular and extracellular metabolites was evaluated by the following assays:

2.3.1 \% DPPH Scavenging Activity

DPPH radical scavenging assay was performed according to the method described by Blois ${ }^{[23]}$ with certain modifications. Ability of microbial extracts of $B$. megaterium to scavenge free radicals was measured by 1, 1- diphenyl-2-picryl hydrazyl. 0.1 $\mathrm{mM}$ DPPH solution was prepared in ethanol. $1 \mathrm{ml}$ of this solution was then further added to $3 \mathrm{ml}$ of microbial extracts, mixed gently and then kept at $37^{\circ} \mathrm{C}$ for half an hour. Absorbance value was recorded at $517 \mathrm{~nm}$ by using spectrophotometer. Ascorbic acid was taken as positive control. Percentage DPPH scavenging effect was measured by the following equation:

$\%$ DPPH rcavenging activity or $\%$ inhibition $=\frac{A_{0}-A_{1}}{A_{0}} \times 100$

Where $A_{0}$ is the Absorbance of control and $A_{1}$ is the Absorbance of test sample

2.3.2 Total Antioxidant Capacity (TAC)

The total antioxidant activity assay was determined by following the standard protocol described by Birben et al. ${ }^{[24]} .1 \mathrm{ml}$ of extract was taken in attesttube, then $1 \mathrm{ml}$ of standard reagent solution $(0.6 \mathrm{M}$ sulphuric acid, $28 \mathrm{mM}$ sodium phosphate, $4 \mathrm{mM}$ ammonium molybdate) was further added to it. The tubes were then kept at $95^{\circ} \mathrm{C}$ for 90 minutes. Absorbance was taken at $695 \mathrm{~nm}$ against blank at room temperature after cooling the reaction mixture. Ascorbic acid was used as positive control. Antioxidant activity was estimated by using following formula:

Total antioxidant capacity $(\%)=\left[\left(\mathrm{Ab}_{\mathrm{c}}-\mathrm{Ab}_{\mathrm{s}}\right) / \mathrm{Ab}_{\mathrm{c}}\right]$ $* 100$;

where $A b_{c}$ is the absorbance of the control and $A b_{s}$ is the absorbance of sample

2.3.3 Total Reductive Capability (TRC)

Total reducing capability was measured by following the standard protocol described by Zhang et $a^{[20]}$. $1 \mathrm{ml}$ of microbial extract was added to 2.5 $\mathrm{ml}(0.2 \mathrm{M})$ Phosphate buffer ( $\mathrm{pH} 6.6)$ and $2.5 \mathrm{ml}$ (1\%) Potassium Ferricyanide. The reaction mixture was kept at $50^{\circ} \mathrm{C}$ for 20 minutes. $2.5 \mathrm{ml}$ of $10 \%$ TriChloro Acetic acid (TCA) was further added and centrifuged at $6500 \mathrm{rpm}$ for 10 minutes. $2.5 \mathrm{ml}$ of upper layer of solution was mixed with equal volumes of distilled water and $500 \mu 1$ of $0.1 \%$ Ferric Chloride. Absorbance value was recorded at 700 $\mathrm{nm}$. Ascorbic acid was taken as reference compound. Increased absorbance values of reaction indicates higher reducing power. The total reductive capability is expressed in terms of $\mathrm{mg}$ equivalent of 


\section{Available online at $w w w . i j r a t . o r g$}

Ascorbic Acid by using the following standard equation:

$\mathrm{Y}=0.5891 \mathrm{x}-0.0959, \mathrm{R}^{2}=0.4963$

\subsection{Synthesis and Characterisation of silver} Nanoparticles

2.4.1 Synthesis of Silver Nanoparticles

Silver Nanoparticles was synthesized in the presence of extracellular metabolites of Bacillus megaterium. Extracellular metabolite was selected on the basis of its relatively better anti-oxidant activity and easy down streaming in comparison to that of extracellular metabolites. Culture supernatant from unaltered media and mixed media was further selected for synthesis of silver Nanoparticles. $2.5 \mathrm{ml}$ of culture supernatant were mixed separately with 1 $\mathrm{mM} \mathrm{AgNO}_{3}$ solution in flasks and then volume make up was done to $50 \mathrm{ml}$ with silver nitrate solution. Flasks were kept on magnetic stirrer for 4 hours along with the control flasks. The reduction of $\mathrm{Ag}^{+}$to $\mathrm{Ag}^{0}$ was confirmed by the colour change of solution from milky white to greyish. The solution was aliquoted in eppendorf tubes and then centrifuged at $7000 \mathrm{rpm}$ for 15 minutes and supernatant was collected. Resuspension and pooling was done by adding $100 \mu \mathrm{l}$ of distilled water. Centrifugation and washing was repeated thrice and greyish pellet of silver nanoparticles was used for further analysis. Pellet was dried at $65^{\circ} \mathrm{C}$, weighed and then resuspended in distilled water at a concentration of $1 \mathrm{mg} / \mathrm{ml}$ and further used for activity analysis and characterization.

2.4.2 Characterization of silver Nanoparticles

The synthesized silver nanoparticles were further characterized by UV-Visible spectroscopy, Dynamic Light Scattering (DLS) and Zeta in order to determine the average particle size and surface charge respectively.

2.4.2.1 UV-Visible spectroscopy

UV-Visible characteristic peak of synthesized silver nanoparticles was monitered by UV-visible spectrophotometer. UV-Visible absorption spectrophotometer was used in the range of 400-600 $\mathrm{nm}$. The absorbance values were recorded for both silver Nanoparticles, $\mathrm{AgNO}_{3}$ solution and distilled water as blank.

2.4.2.2 DLS and Zeta potential

Average size, polydispersity index and zeta potential of nanoparticles were measured by dynamic light scattering (DLS) using Malvern Zetasizer Nanos ZS90 (Malvern Instruments, UK) by using the method devised by Kumar et al. ${ }^{[25]}$ with slight modifications. $50 \mu \mathrm{l}$ of synthesised silver nanoparticles were dispersed in double distilled water to make up the volume up to $1 \mathrm{ml}$ and readings were noted at $25^{\circ} \mathrm{C}$.

\section{RESULTS AND DISCUSSION}

The results of anti-oxidant analysis of metabolites and synthesised silver nanoaparticles are summarized under the following headings:

3.1. Antioxidant Activity analysis of extracellular and intracellular metabolites of $B$. megaterium in unaltered and mixed media Antioxidant analysis of extracellular, intracellular metabolites and media were evaluated as a function of DPPH radical scavenging activity, Total anti-oxidant Capacity and Total reductive capability. The results are summarised as follows:

\subsubsection{DPPH radical scavenging activity}

Graph 1 indicates the percentage radical scavenging activity of cell free supernatant, biomass suspension and nutrient broth of both unaltered and mixed media. It could be inferred that the supernatant, i.e. extracellular metabolites collected from mixed media (94\%) have higher scavenging activity in comparison to that of unaltered media.

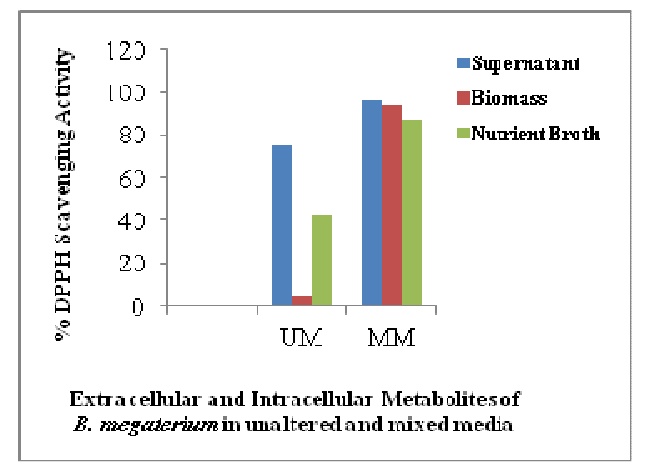

Graph 1: \% DPPH radical scavenging activity of intracellular and extracellular metabolites of $B$. megaterium in unaltered and mixed media

\subsubsection{Total Anti-oxidant Capacity}

Graph 2 indicates the Total Anti-oxidant Capacity of cell free supernatant, biomass suspension and nutrient broth of both unaltered and mixed media. It could be inferred from the graph that the extracellular metabolites secreted in mixed media have higher Total Anti-oxidant Capacity (76.37\%) in comparison to that of unaltered media $(25.71 \%)$. 


\section{Available online at www.ijrat.org}

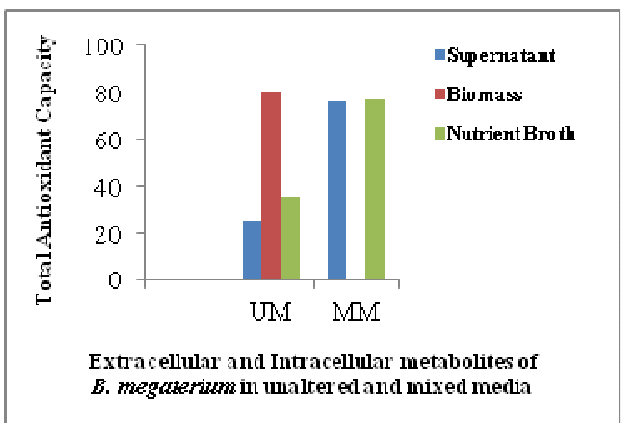

Graph 2: \% Total antioxidant capacity of intracellular and extracellular metabolites of $B$. megaterium in unaltered and mixed media

\subsubsection{Total Reductive Capability}

Graph 3 indicates the total reductive capability of cell free supernatant, biomass suspension and nutrient broth of both unaltered and mixed media. It could be inferred from the graph that the extracellular metabolites secreted in unaltered media have higher total reductive capability $(0.3316 \quad \mathrm{mg}$ ascorbic acid equivalent) in comparison to that of mixed media ( $0.213 \mathrm{mg}$ ascorbic acid equivalent).

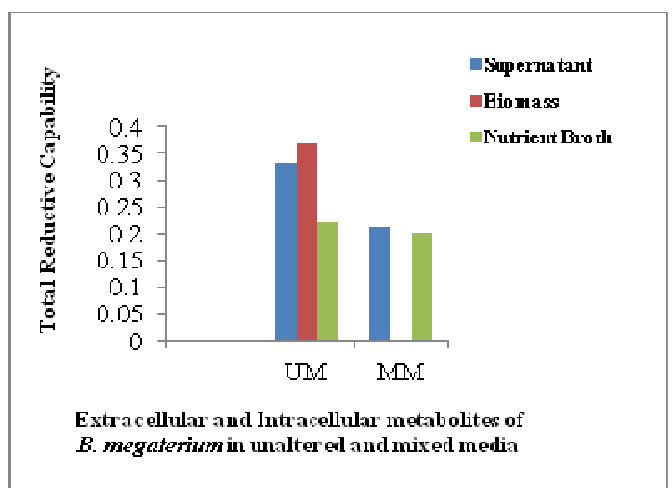

Graph 3: \% Total Reductive Capability of intracellular and extracellular metabolites of $B$. megaterium in unaltered and mixed media

3.2 Synthesis and Characterisation of silver nanoparticles in presence of extracellular microbial metabolites

Silver Nanoparticles were synthesised by using media supernatant of unaltered and mixed media $\left(37^{\circ} \mathrm{C}, \mathrm{pH} 7\right)$. Table 3 summarises the yield of Nanoparticles from $B$. megaterium

Table 3 : AgNps synthesised from Unaltered and Mixed Media (B. megaterium)

\begin{tabular}{|l|l|l|}
\hline S. No. & Extracellular Metabolites & $\begin{array}{l}\text { Yield } \\
\text { (in mg/ml) }\end{array}$ \\
\hline
\end{tabular}

\begin{tabular}{|l|l|l|}
\hline 1 & $\mathrm{UM}, \mathrm{pH} 7,37^{\circ} \mathrm{C}$ & 0.04 \\
\hline 2 & $\mathrm{MM}, \mathrm{pH} 7,37^{\circ} \mathrm{C}$ & 0.64 \\
\hline
\end{tabular}

\subsubsection{Characterisation of Silver Nanoparticles}

\subsubsection{UV-Vis Spectroscopy}

Synthesis of the AgNPs in aqueous solution was monitored by measuring the absorbance at a wavelength range of 405-630 nm. It was observed that solution of silver nitrate turned dark brown on addition of microbial extracts after an incubation period of 4 hours on magnetic stirrer. This indicates the formation of AgNPs. This occurs because of change in Surface Plasmon Resonance (SPR) when positive ions of silver are converted into its corresponding nano atoms ${ }^{[26]}$.

Graph 4 indicates the surface Plasmon resonance of $\mathrm{AgNp}$ synthesised from extracellular metabolites collected from unaltered media of $B$. megaterium. Steeper peak at $405 \mathrm{~nm}$ indicates that majority of the particles are of smaller size range which could be further confirmed by DLS study.

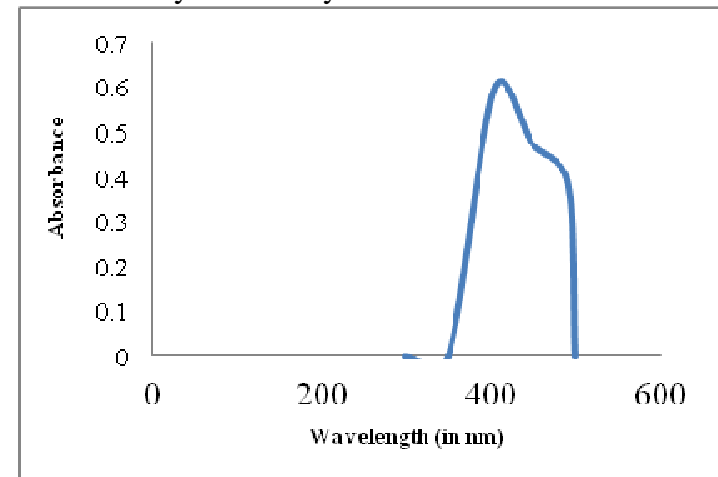

Graph 4: UV-Vis Surface Plasmon Resonance of AgNp synthesised from UM supernatant of $B$. megaterium

Graph 5 indicates the surface Plasmon resonance of $\mathrm{AgNp}$ synthesised from extracellular metabolites collected from mixed media of $B$. megaterium. A sharp Surface plasmon resonance (SPR) peak was observed at the wavelength $405 \mathrm{~nm}$ that confirmed the formation of silver nanoparticles. Hence, it can be concluded that MM supernatant posses the property to reduce the silver particles into AgNps. 


\section{Available online at www.ijrat.org}

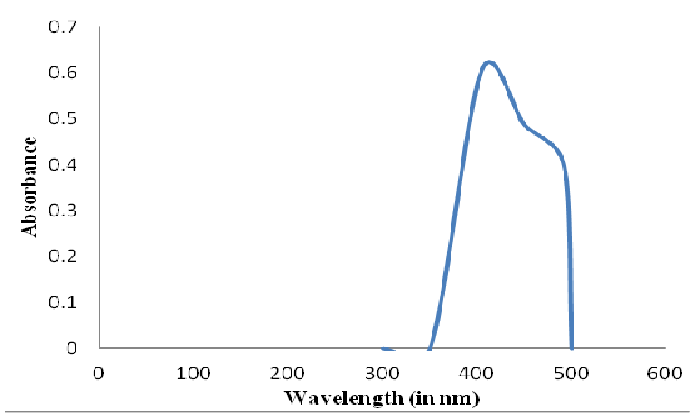

Graph 5: UV-Vis Surface Plasmon Resonance of AgNp synthesised from MM supernatant of B. megaterium

3.2.1.1 DLS and Zeta potential

Graph 6 indicates DLS measurement of $\mathrm{AgNp}$ synthesised from media supernatant of unaltered media of $B$. megaterium. The particles are in the size range of $164.2 \mathrm{~nm}-396.1 \mathrm{~nm}$. It could be observed that majority of particles were of $220 \mathrm{~nm}$.

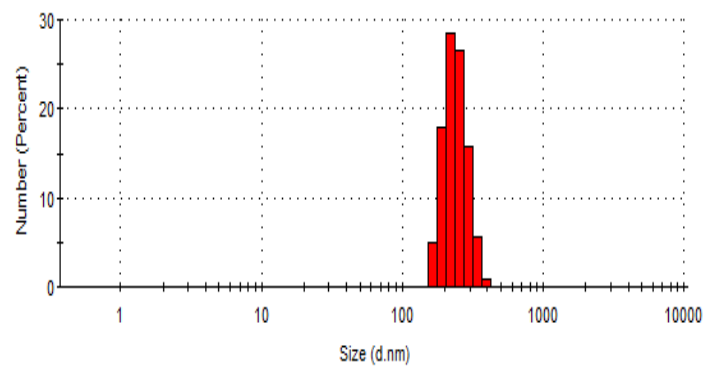

Graph 6: DLS measurement of AgNp synthesised from unaltered media supernatant of B. megaterium

Graph 7 indicates zeta potential measurement of $\mathrm{AgNp}$ synthesised from media supernatant of unaltered media of $B$. megaterium. The zeta value is found to be $-28.8 \mathrm{mV}$.

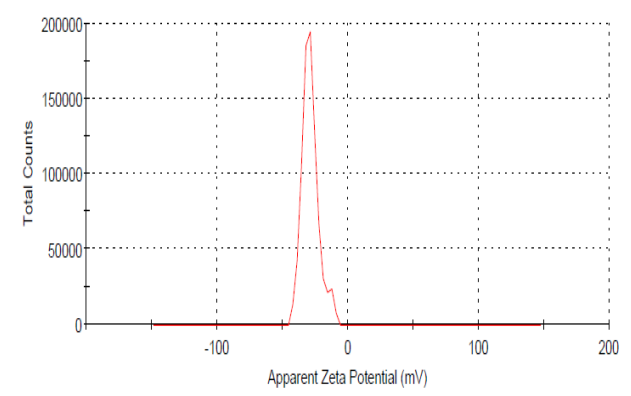

Graph 7: Zeta potential measurement of AgNp synthesised from unaltered media supernatant of B. megaterium

Graph 8 indicates DLS measurement of $\mathrm{AgNp}$ synthesised from media supernatant of mixed media of $\quad$ B. megaterium. The particles are in the size range of $91.28-255 \mathrm{~nm}$. The majority of particles were found to be of $122.4 \mathrm{~nm}$. The decrease in size could be because of the metabolites secreted in mixed media which may serve as capping agents for the particles that help them to remain segregated. Hence, Mixed Media was further selected for $\mathrm{pH}$ and temperature variation.

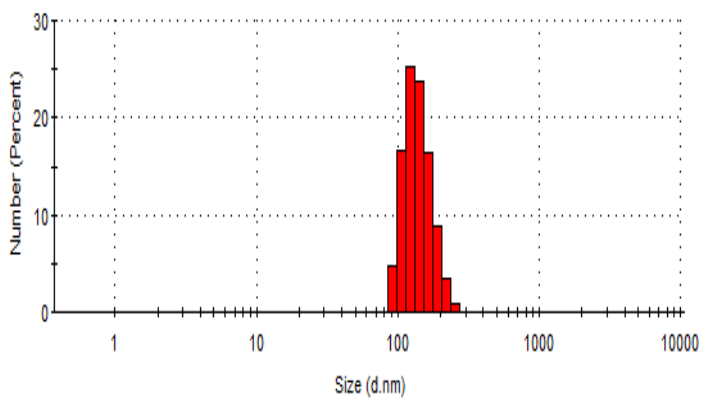

Graph 8 DLS measurement of AgNp synthesised from mixed media supernatant of $B$. megaterium

Graph 9 indicates zeta potential measurement of AgNp synthesised from media supernatant of mixed media of $B$. megaterium. The zeta value was found to be $-19.2 \mathrm{mV}$. Though the zeta value was low in case of mixed but since much small sized particles were obtained in comparison to unaltered media, hence mixed media was selected for further study. The possible reason for decrease in Zeta value may be due to the thinner coating of extra cellular metabolites secreted in mixed media on the surface of AgNp. The results are in agreement with a previous study done by Skandalis et al ${ }^{[27]}$.

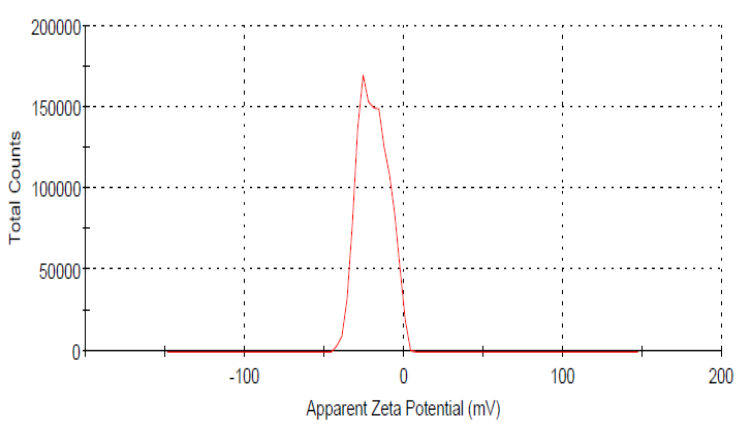

Graph 9 Zeta potential measurement of AgNp synthesised from mixed media supernatant of $B$. megaterium

\subsection{Anti-oxidant activity analysis of synthesised} silver nanoparticles

3.3.1 DPPH radical scavenging activity for silver nanoparticles

DPPH radical scavenging activity of silver nanoparticles synthesized from extracellular metabolites of $B$. megaterium of unaltered and mixed media formulations (UM and MM) are indicated in Graph 10. Silver Nanoparticles synthesized from MM was identified to have the highest percentage of DPPH radical scavenging 
activity. Since, the results are in accordance by the work done by Nagaich ${ }^{[28]}$. Hence, it could be inferred that MM was the best media formulation for the production of silver nanoparticles with good scavenging ability.

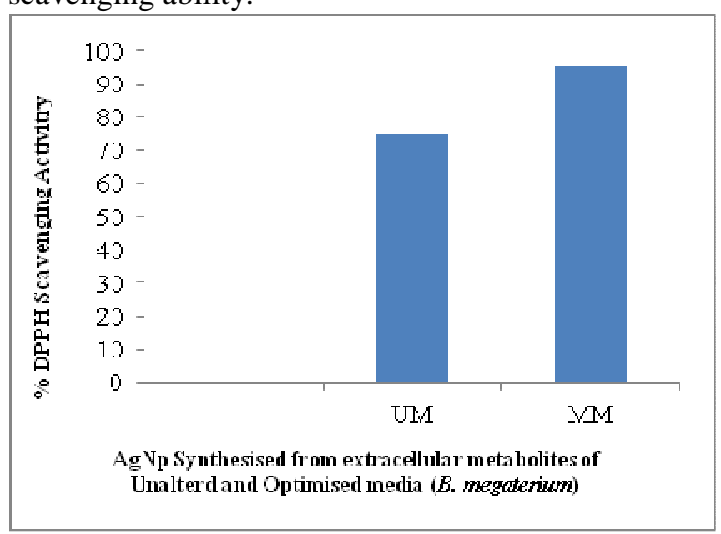

Graph 10: DPPH radical scavenging activity for AgNp synthesised from extracellular metabolites of B. megaterium (Unaltered and Mixed Media)

\subsubsection{Total Anti-oxidant Capacity}

Total Anti-oxidant Capacity of silver nanoparticles synthesized from extracellular metabolites of $B$. megaterium of unaltered and mixed media formulations (UM and MM) are indicated in Graph 11. Silver Nanoparticles synthesized from extracellular metabolites of MM $(98.03 \%)$ have better total anti-oxidant Capacity in comparison to that of Unaltered Media (88.29\%).

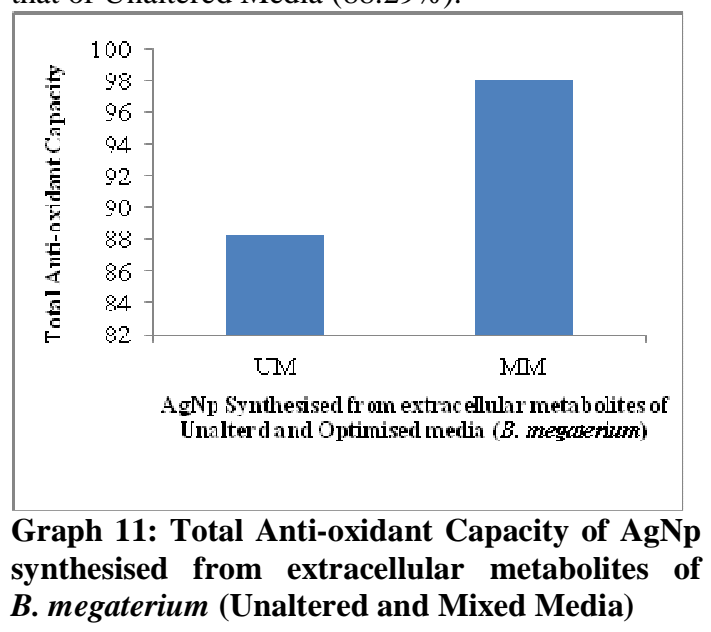

\subsubsection{Total Reductive Capability}

Total Reductive Capability of silver nanoparticles synthesized from extracellular metabolites of $B$. megaterium of unaltered and mixed media formulations (UM and MM) are indicated in Graph 12. Silver Nanoparticles synthesized from extracellular metabolites of unaltered media have higher reductive capability in comparison to that of mixed media.

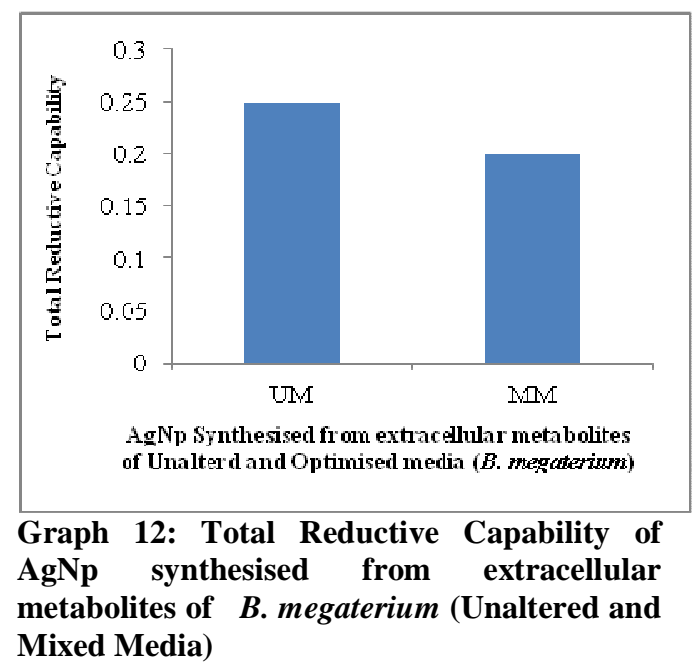

\section{CONCLUSION}

To the best of our knowledge, the effect of extracellular metabolites released by $B$. megaterium in different media composition was studied on the size range and stability of silver nano particles. The results show that extracellular metabolites released in mixed media served as better capping and reducing agents thus reducing the particle size and enhancing the stability in comparison to those metabolites released in unaltered media. Yield of silver Nanoparticles from mixed media supernatant was found to be $0.64 \mathrm{mg} / \mathrm{ml}$. Absorbance maxima for synthesised silver nanoparticles from unaltered and mixed media was found to be at $405 \mathrm{~nm}$. In case of unaltered media supernatant, the particles are in the size range of $164.2 \mathrm{~nm}-396.1 \mathrm{~nm}$. In case of mixed media supernatant, the particles are in the size range of $91.28-255 \mathrm{~nm}$. The zeta value was found to be $-28.8 \mathrm{mV}$ and $-19.2 \mathrm{mV}$ for unaltered and mixed media respectively. Silver Nanoparticles synthesized from extracellular metabolites of Mixed Media (98.03\%) have better total anti-oxidant Capacity in comparison to that of Unaltered Media $(88.29 \%)$. Similar results were also observed with DPPH radical scavenging activity.

Extracellular metabolite showed higher anti-oxidant potential in comparison to the biomass suspension. Silver Nanoparticles synthesised from mixed media supernatant have smaller size as well as higher antioxidant potential in comparison to the unaltered media. This may be due to the enhanced metabolite secretion in mixed media which may serve as capping agents thus maintaining the synthesised nanoparticles in monodisperse form.

\section{ACKNOWLEDGEMENT}

The authors would like to acknowledge Dr. Sanjay Swain, Research Officer, BIT Mesra for providing assistance and facilities for DLS and Zeta analysis. They would also thank Chairperson of the University, Dr. Aseem Chauhan and Honourable 


\section{Available online at www.ijrat.org}

Vice-Chancellor, Lt. Gen V. K. Sharma for providing suitable infrastructure and research facilities.

\section{CONFLICT OF INTEREST STATEMENT}

The authors declare no conflict of interest.

\section{REFERENCES}

[1] Halliwell B and Gutteridge JMC (1984). Oxygen toxicity, oxygen radicals, transition metals and disease. Biochemical Journal 219 (1): 1-4.

[2] Battino $\mathrm{M}$, Bullon $\mathrm{P}$, Wilson $\mathrm{M}$ and Newman $\mathrm{H}$ (1999). Oxidative injury and challenge of antioxidants to free radicals and reactive oxygen species. Critical rev. Oral biol. Medi. 10: 458-476.

[3] Meghashri S, Kumar HV and Gopal S (2010). Antioxidant properties of a novel flavonoid from leaves of Leucas aspera. Food Chemistry 122 (1): 105-110.

[4] Yoghiapiscessa D, Batubara I and Wahyudi AT (2016). Antimicrobial and Antioxidant Activities of Bacterial Extracts from Marine Bacteria Associated with Sponge Stylotella sp. American Journal of Biochemistry and Biotechnology: 01-10.

[5] Gupta C, Prakash D and Gupta S (2013). Functional Foods Enhanced with Microbial Antioxidants. Academic Journal of Nutrition 2 (2): 10-18.

[6] Kumar PS, Al-dhabi NA, Duraipandiyan V, Balachandran C, Kumar PP and Ignacimuthu S (2014). In vitro antimicrobial, antioxidant and cytotoxic properties of Streptomyces lavendulae strain SCA5. Bmc microbiology 14: 291.

[7] Kaur V, Bera MB, Panesar PS, Chopra HK (2013). Production and characterization of exopolysaccharide produced by Alcaligenes faecalis B14 isolated from indigenous soil. International journal of biotechnology and bioengineering research 4 (4): 365-74.

[8] Rai M, Yadav A, Gade A (2009). Silver nanoparticles as a new generation of antimicrobials. Biotechnol Adv 27:76-83.

[9] Sondi I, Salopek-Sondi B (2004). Silver nanoparticles as antimicrobial agent: a case study on E. coli as a model for Gram-negative bacteria. J Colloid Interf Sci 275: 177-82.

[10] Jo YK, Kim BH, Jung G (2009). Antifungal activity of silver ions and nanoparticles on phytopathogenic fungi. Plant Dis 93: 1037-43.

[11] Kim JS, Kuk E, Yu KN, Kim JH, Park SJ, Lee HJ, Kim SH, Park YK, Park YH, Hwang CY, Kim YK, Lee YS, Jeong DH and Cho MH (2007). Antimicrobial effects of silver nanoparticles. Nanomedicine 3: 95-101.

[12] Lara HH, Ayala-Nunez NV, Ixtepan-Turrent L, Rodriguez- Padilla C (2010). Mode of antiviral action of silver Nanoparticles against HIV-1. J Nanobiotechnology 8: 1 .

[13] Lu L, Sun RW, Chen R, Hui CK, Ho CM, Luk JM, Lau GK, Che CM (2008). Silver nanoparticles inhibit hepatitis B virus replication. Antivir Ther 13: 253-62.

[14] Baram-Pinto D, Shukla S, Perkas N, Gedanken A, Sarid R (2009). Inhibition of herpes simplex virus type 1 infection by silver nanoparticles capped with mercaptoethane sulfonate. Bioconjug Chem 20: 1497502.

[15] Wong KKY, Cheung SOF, Huang LM, Niu J, Tao C, Ho CM, Che CM and Tam PK (2009). Further evidence of the anti-inflammatory effects of silver nanoparticles. Chemmedchem 4: 1129-35.

[16] Nadworny PL, Wang J, Tredget EE and Burrell RE (2008). Anti-inflammatory activity of nanocrystalline silver in a porcine contact dermatitis model. Nanomedicine 4: 241-51.

[17] Sun Y, Yin Y, Mayers BT, Herricks T and Xia Y (2002). Uniform silver nanowires synthesis by reducing $\mathrm{AgNO}_{3}$ with ethylene glycol in the presence of seeds and poly (vinyl pyrrolidone). Chem Mater 14: 47364745.

[18] Yin B, Ma H, Wang S and Chen S (2003). Electrochemical synthesis of silver nanoparticles under protection of poly (n-vinylpyrrolidone). J Phys Chem B 107: 8898-904.

[19] Callegari A, Tonti D and Chergui M (2003). Photochemically grown silver nanoparticles with wavelength-controlled size and shape. Nano Lett 3: 1565-68.

[20] Zhang DM, Xu HG, Wang L, Li YJ, Sun PH, Wu XM, Wang GJ, Chen WM and Ye WC (2015). Betulinic acid and its derivatives as potential antitumor agents. Med. Res. Rev. 35, 1127-1155.

[21] Swami A, Selvakannan PR, Pasricha R and Sastry M (2004). One-step synthesis of ordered two-dimensional assemblies of silver nanoparticles by the spontaneous reduction of silver ions by pentadecylphenol langmuir monolayers. J Phys Chem B 108: 19269-75.

[22] Naik RR, Stringer SJ, Agarwal G, Jones S and Stone MO (2002). Peptide templates for nanoparticle synthesis derived from polymerase chain reaction-driven phage display. Adv Funct Mater 14:25-30.

[23] Blois MS (1958). Antioxidant determinations by the use of a stable free radical. Nature 29: 1199-1200.

[24] Birben E, Sahiner UM, Sackesen C, Erzurum S and Kalayci O (2012). Oxidative Stress and Antioxidant Defense. World Allergy Organ J 5 (1): 9-19.

[25] Kumar N, Salar RK, Prasad M and Ranjan K (2018). Synthesis, characterization and anticancer activity of vincristine loaded folic acid-chitosan conjugated nanoparticles on NCI-H460 non-small cell lung cancer cell line. Egyptian Journal of Basic and Applied Sciences 5: 87-99.

[26] Mittal AK, Chisti Y and Banerjee UC (2013). Synthesis of metallic Nanoparticles using plant extracts. Biotechnol adv. 31 (2): 346-56.

[27] Skandalis N, Dimopoulou A, Georgopoulou A, Gallios N, Papadopoulos D, Tsipas D, Theologidis I, Michailidis N and Chatzinikolaidou M (2017). The effect of silver nanoparticles size, produced using plant 
International Journal of Research in Advent Technology, Vol.7, No.4, April 2019 E-ISSN: 2321-9637

\section{Available online at www.ijrat.org}

extract from Arbutus unedo, on their antibacterial efficacy. Nanomaterials 7 (178): 1-14.

[28] Nagaich U, Gulati N and Chauhan S (2016). Antioxidant and Antibacterial Potential of Silver Nanoparticles: Biogenic Synthesis Utilizing Apple Extract. Journal of Pharmaceutics 7141523: 1-8. 